\title{
RETRACTED ARTICLE: Astrocytes Regulate Differentiation and Glutamate Uptake of Glioma Stem Cells via Formyl Peptide Receptor
}

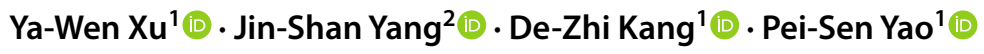

Received: 20 March 2020 / Accepted: 25 May 2020 / Published online: 30 May 2020

(c) Springer Science+Business Media, LLC, part of Springer Nature 2020

The authors have retracted this article because in follow up expressions the EAAT1 expression on cytomembranes of glioma stem cells could not be replicated. Additionally, the authors did not have permission to utilize and publish the data regarding the EAAT1 antibody. All authors agree with this retraction.
The online version of this article contains the full text of the retracted article as Supplementary Information.

Supplementary Information The online version contains supplementary material available at https://doi.org/10.1007/s10571-020-00886-3.

Publisher's Note Springer Nature remains neutral with regard to jurisdictional claims in published maps and institutional affiliations.

Ya-Wen $\mathrm{Xu}$ and Jin-Shan Yang equally contributed to this article.

De-Zhi Kang

kdzy99988@163.com

$\triangle$ Pei-Sen Yao

peisen.yao@163.com

1 Department of Neurosurgery, First Affiliated Hospital of Fujian Medical University, NO. 20 Chazhong Road, Taijiang District, Fuzhou 350004, Fujian, China

2 Department of Neurology, First Affiliated Hospital of Fujian Medical University, Fuzhou, China 